\title{
Reciprocal Complementary Wiener Numbers of Non-Caterpillars
}

\author{
Yanli Zhu, Fuyi Wei, Feng Li \\ Department of Applied Mathematics, South China Agricultural University, \\ Guangzhou, China \\ Email: yanlizhu130@tom.com
}

Received 14 January 2016; accepted 23 February 2016; published 26 February 2016

Copyright (C) 2016 by authors and Scientific Research Publishing Inc.

This work is licensed under the Creative Commons Attribution International License (CC BY).

http://creativecommons.org/licenses/by/4.0/

\section{(c) (i) Open Access}

\section{Abstract}

The reciprocal complementary Wiener number of a connected graph $G$ is defined as

$$
R C W(G)=\sum_{\{u, v\} \subseteq V(G)} \frac{1}{d+1-d(u, v \mid G)}
$$

where $V(G)$ is the vertex set. $d(u, v \mid G)$ is the distance between vertices $u$ and $v$, and $d$ is the diameter of $G$. A tree is known as a caterpillar if the removal of all pendant vertices makes it as a path. Otherwise, it is called a non-caterpillar. Among all $n$-vertex non-caterpillars with given diameter $d$, we obtain the unique tree with minimum reciprocal complementary Wiener number, where $4 \leq d \leq n-3$. We also determine the $n$-vertex non-caterpillars with the smallest, the second smallest and the third smallest reciprocal complementary Wiener numbers.

\section{Keywords}

Reciprocal Complementary Wiener Number, Wiener Number, Caterpillar

\section{Introduction}

The Wiener number was one of the oldest topological indices, which was introduced by Harry Wiener in 1947. About the recent reviews on matrices and topological indices related to Wiener number, refer to [1]-[4]. The RCW number is one of the hotest additions in the family of such descriptors. The notion of RCW number was first put forward by Ivanciuc and its applications were discussed in [5]-[8]. 
Let $G$ be a simple connected graph with vertex set $V(G)$. For two vertices $u, v \in V(G)$, let $d(u, v \mid G)$ denote the distance between $u$ and $v$ in $G$. Then, the RCW number of $G$ is defined by

$$
R C W(G)=\sum_{\{u, v\} \subseteq V(G)} \frac{1}{d+1-d(u, v \mid G)}
$$

where $d$ is the diameter and the summation goes over all unordered pairs of distinct vertices of $G$. Some properties of the RCW number have been obtained in [9] [10].

A tree is called a caterpillar if the removal of all pendant vertices makes it as a path. Otherwise, it is called a non-caterpillar.

For integers $n$ and $d$ satisfying $4 \leq d \leq n-3$, let $N_{n, d, i}$ be the tree obtained from the path $P_{d+1}$ labelled as $v_{0}, v_{1}, \cdots, v_{d}$ by attaching the path $P_{2}$ and $n-d-3$ pendant vertices to vertex $v_{i}$ for $2 \leq i \leq\left\lfloor\frac{d}{2}\right\rfloor$ (see Figure 1). Let $N_{n, d}=N_{n, d,\left[\frac{d}{2}\right.}$.

In this paper, we show that among all $n$-vertex non-caterpillars with given diameter $d, N_{n, d}$ is the unique tree with minimum RCW number where $4 \leq d \leq n-3$. Furthermore, we determine the non-caterpillars with the smallest, the second smallest and the third smallest RCW numbers.

\section{RCW Numbers of Non-Caterpillars}

All $n$-vertex trees with diameter 2, 3, n-2 and $n-1$ are caterpillars. Let $n$ and $d$ be integers with $n \geq 7$ and $4 \leq d \leq n-3$. Let $\mathbf{N C}(n, d)$ be the class of non-caterpillars with $n$ vertices and diameter $d$. Let $\mathcal{N C}(n, d)$ be the class of non-caterpillars obtained by attaching the stars $s_{n_{1}}, \cdots, s_{n_{t}}$ at their centers and $s=n-d-1-\sum_{i=1}^{t} n_{i}$ pendant vertices to one center (fixed if it is bicentral) of the path $P_{d+1}$, where $t \geq 1, s \geq 0$ and $n_{i} \geq 2$ for $i=1,2, \cdots, t$ (see Figure 2). Recall that $N_{n, d}=N_{n, d,\left\lfloor\frac{d}{2}\right\rfloor}$. Obviously, $N_{n, d} \in \mathcal{N C}(n, d) \subseteq \mathbf{N C}(n, d)$ and $\mathcal{N C}(n, n-3)=\left\{N_{n, n-3}\right\}$.

Let $T$ be a tree. For $u \in V(T)$ and $A \subseteq V(T)$, let $\delta_{T}(u)$ be the degree of $u$ in $T$ and $d_{T}(u, A)$ be the sum of all distances from $u$ to the vertices in $A$, i.e., $d_{T}(u, A)=\sum_{v \in A} d_{T}(u, v)$. Here and in the following $d_{T}(u, v)$ denotes the distance between vertices $u$ and $v$ in $T$.

Lemma 1 Let $T$ be a tree with minimum $R C W$ number in $\mathrm{NC}(n, d)$, where $4 \leq d \leq n-3$. Then, $T \in \mathcal{N C}(n, d)$.

Proof. Suppose that $T \in \mathbf{N C}(n, d) \backslash \mathcal{N C}(n, d)$. Let $P(T)=v_{0} v_{1} \cdots v_{d}$ be a diametral path of $T$. If $d$ is odd, we require that $\delta_{T}\left(v_{\left\lfloor\frac{d}{2}\right\rfloor}\right) \geq \delta_{T}\left(v_{\left\lceil\frac{d}{2}\right\rceil}\right)$. Then at least one of $v_{2}, \cdots, v_{d-2}$ has degree at least three. There are two cases.

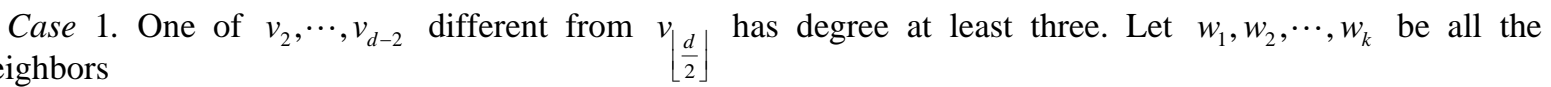
outside $P(T)$ except those of $v_{\left\lfloor\frac{d}{2}\right\rfloor}$, where $w_{i}$ is a neighbor of $v_{i^{\prime}} \in V(P(T))$. Let $T_{i}$ be the subtree of $T-v_{i^{\prime}}$ containing $w_{i} . T^{*}$ be the tree formed from $T$ by deleting edges $w_{i} v_{i^{\prime}}$ and adding edges $w_{i} v_{\left\lfloor\frac{d}{2}\right\rfloor}$ for

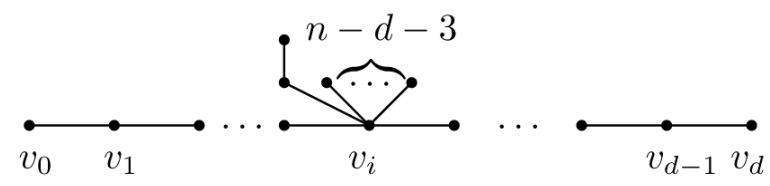

Figure 1. The tree $N_{n, d, i}$. 


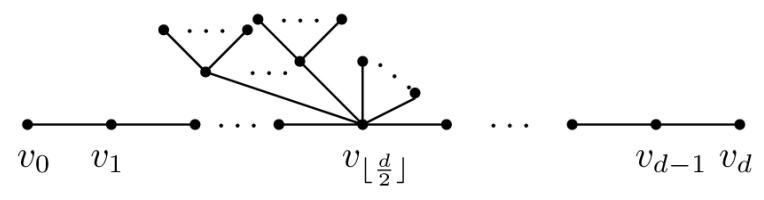

Figure 2. The tree $N C(n, d)$.

all $i=1,2, \cdots, k$. Obviously, $T^{*} \in \mathbf{N C}(n, d)$. Let $A=V(P(T))$ and $B=V(T) \backslash A$. It is easily seen that

$$
\begin{aligned}
R C W(T)-R C W\left(T^{*}\right)= & \sum_{u \in B, v \in A}\left[\frac{1}{d+1-d_{T}(u, v)}-\frac{1}{d+1-d_{T^{*}}(u, v)}\right] \\
& +\sum_{\{u, v\} \leq B}\left[\frac{1}{d+1-d_{T}(u, v)}-\frac{1}{d+1-d_{T^{*}}(u, v)}\right] \\
\geq & \sum_{\substack{u \in V\left(T_{i}\right) v \in A \\
1 \leq i \leq k}}\left[\frac{1}{d+1-d_{T}(u, v)}-\frac{1}{d+1-d_{T^{*}}(u, v)}\right] \\
& +\sum_{\substack{u \in V\left(T_{i}, v \in V\left(T_{j}\right) \\
1 \leq i<j \leq k\right.}}\left[\frac{1}{d+1-d_{T}(u, v)}-\frac{1}{d+1-d_{T^{*}}(u, v)}\right]
\end{aligned}
$$

with equality if and only if $\delta_{T}\left(v_{\left\lfloor\frac{d}{2}\right\rfloor}\right)=2$. Since $i^{\prime} \neq\left\lfloor\frac{d}{2}\right\rfloor, \quad d_{T}\left(u, v_{i^{\prime}}\right)=d_{T^{*}}\left(u, v_{\left\lfloor\frac{d}{2}\right\rfloor}\right)$ for $u \in V\left(T_{i}\right)$ and $v_{i^{\prime}} \in A$ with $1 \leq i \leq k$. We get

$$
\begin{aligned}
\sum_{v \in A} \frac{1}{d+1-d_{T}(u, v)} & =\sum_{v \in A} \frac{1}{d+1-d_{T}\left(u, v_{i^{\prime}}\right)-d_{T}\left(v_{i^{\prime}}, v\right)} \\
& =\sum_{k=1}^{i^{\prime}} \frac{1}{d+1-d_{T}\left(u, v_{i^{\prime}}\right)-k}+\sum_{k=1}^{d-i^{\prime}} \frac{1}{d+1-d_{T}\left(u, v_{i^{\prime}}\right)-k} \\
& \geq \sum_{k=1}^{\left\lfloor\frac{d}{2}\right\rfloor} \frac{1}{\left.d+1-d_{T^{*}}\left(u, v_{\left\lfloor\frac{d}{2}\right\rfloor}\right\rfloor\right)-k}+\sum_{k=1}^{\left.\frac{d}{2}\right]} \frac{1}{\left.d+1-d_{T^{*}}\left(u, v_{\left\lfloor\frac{d}{2}\right\rfloor}\right\rfloor\right)-k} \\
& =\sum_{v \in A} \frac{1}{\left.\left.d+1-d_{T^{*}}\left(u, v_{\left\lfloor\frac{d}{2}\right\rfloor}\right\rfloor\right)-d_{T^{*}}\left(v_{\left\lfloor\frac{d}{2}\right\rfloor}\right\rfloor\right)}=\sum_{v \in A} \frac{1}{d+1-d_{T^{*}}(u, v)} .
\end{aligned}
$$

Then

$$
\begin{aligned}
& \sum_{\substack{u \in V \in\left(T_{i}\right) v \in A \\
1 \leq i \leq k}}\left[\frac{1}{d+1-d_{T}(u, v)}-\frac{1}{d+1-d_{T^{*}}(u, v)}\right] \\
& =\sum_{\substack{u \in V\left(T_{i}\right) \\
1 \leq i \leq k}}\left[\sum_{v \in A} \frac{1}{d+1-d_{T}(u, v)}-\sum_{v \in A} \frac{1}{d+1-d_{T^{*}}(u, v)}\right] \geq 0
\end{aligned}
$$

with equality if and only if $i^{\prime}=\left\lceil\frac{d}{2}\right\rceil$ (which is only possible for odd number $d$ ). But $\delta_{T}\left(v_{\left\lfloor\frac{d}{2}\right\rfloor}\right) \geq \delta_{T}\left(v_{\left\lceil\frac{d}{2}\right\rceil}\right)$, and 
thus if $\delta_{T}\left(v_{\left.\mid \frac{d}{2}\right\rfloor}\right)=2$ then $\delta_{T}\left(v_{\left\lceil\frac{d}{2}\right\rceil}\right)=2$. So $i^{\prime} \neq\left\lceil\frac{d}{2}\right\rceil$ for $i=1,2, \cdots, k$. Thus

$$
\begin{aligned}
& R C W(T)-R C W\left(T^{*}\right) \\
& >\sum_{\substack{u \in V\left(T_{i}\right), v \in V\left(T_{j}\right) \\
1 \leq i<j \leq k}}\left[\frac{1}{d+1-d_{T}(u, v)}-\frac{1}{d+1-d_{T^{*}}(u, v)}\right] \geq 0,
\end{aligned}
$$

since $d_{T}(u, v) \geq d_{T^{*}}(u, v)$ for $u \in V\left(T_{i}\right), v \in V\left(T_{j}\right) \quad(1 \leq i<j \leq k)$. It follows that $R C W(T)>R C W\left(T^{*}\right)$. This is a contradiction.

Case 2. Any verter $v_{i}$ with $i=2,3, \cdots, d-2$ and $i \neq\left\lfloor\frac{d}{2}\right\rfloor$ has degree two. Obviously, $\delta_{T}\left(v_{\left\lfloor\frac{d}{2}\right\rfloor}\right\rfloor \geq 3$. Let $x y z \cdots v_{\left\lfloor\frac{d}{2}\right\rfloor}$ be the (unique) path from $x$ to $\quad v_{\left\lfloor\frac{d}{2}\right\rfloor}$ in $T$ such that $d_{T}\left(x, v_{\left\lfloor\frac{d}{2}\right\rfloor}\right)=\max _{u \in V(T) \backslash V(P(T))} d_{T}\left(u, v_{\left\lfloor\frac{d}{2}\right\rfloor}\right)$. Since $T \notin \mathcal{N C}(n, d)$, we have $d_{T}\left(x, v_{\left\lfloor\frac{d}{2}\right\rfloor}\right) \geq 3$. Let $x_{1}, \cdots, x_{r}, z$ be the neighbors of $y$ in $T$, where $x_{1}=x$ and $r \geq 1$. Let $T^{* *}$ be the tree obtained from $T$ by deleting edges $y x_{i}$ and adding edges $z x_{i}$ for all $i=1,2, \cdots, r$. Then $T^{* *} \in \mathbf{N C}(n, d)$. Let $N_{y}=\left\{x_{1}, \cdots, x_{r}\right\}, C=V(T) \backslash N_{y}$. Since $d_{T}(u, v)=d_{T^{* *}}(u, v)+1$ for $u \in N_{y}, v \in C \backslash\{y, z\}$, we get

$$
\begin{aligned}
& R C W(T)-R C W\left(T^{* *}\right) \\
= & \sum_{u \in N_{y}, v \in C}\left[\frac{1}{d+1-d_{T}(u, v)}-\frac{1}{d+1-d_{T^{*}}(u, v)}\right] \\
= & \frac{r-1}{d+1-1}+\frac{r-1}{d+1-2}+\sum_{u \in N_{y}, v \in C \backslash\{y, z\}} \frac{1}{d+1-d_{T}(u, v)} \\
& -\frac{r-1}{d+1-1}-\frac{r-1}{d+1-2}-\sum_{u \in N_{y}, v \in C \backslash\{y, z\}} \frac{1}{d+1-d_{T^{*}}(u, v)} \\
> & 0 .
\end{aligned}
$$

This is a contradiction.

By combining Cases 1 and 2, we find that $T \in \mathbf{N C}(n, d) \backslash \mathcal{N C}(n, d)$ is impossible. The result follows.

Lemma 2 Let $T \in \mathcal{N C}(n, d)$ with $4 \leq d \leq n-3$. Then

$$
R C W(T) \geq R C W\left(N_{n, d}\right)
$$

with equality if and only if $T=N_{n, d}$.

Proof. Let $T$ be a tree with the minimum RCW number in $\mathcal{N C}(n, d)$. Let $P(T)=v_{0} v_{1} \cdots v_{d}$ be a diametral path of $T$.

Suppose that there is a vertex $u \in V(T) \backslash V(P(T))$ with $\delta_{T}(u) \geq 3$. Let $u_{1}, u_{2}, \cdots, u_{s}$ be the neighbors of $u$ different from $v_{\left.\right|^{d}}$ in $T$, where $s \geq 2$. Clearly, $u_{i}$ are pendant vertices for $i=1,2, \cdots, s$. Let $T^{\prime}$ be the tree obtained from $T$ by deleting edges $u u_{i}$ and adding edges $v_{\left\lfloor\frac{d}{2}\right.} u_{i}$ for $i=2, \cdots, s$. Obviously, $T^{\prime} \in \mathcal{N C}(n, d)$.

Let $N_{u}=\left\{u_{2}, \cdots, u_{s}\right\}, D=V(T) \backslash N_{u}$, and $K=\left\{u, u_{1}, v_{\left\lfloor\frac{d}{2}\right\rfloor}, v_{\left\lfloor\frac{d}{2}\right\rfloor-1}\right\}$. Since $d_{T}(u, v)=d_{T^{\prime}}(u, v)+1$ for $u \in N_{u}, v \in D \backslash K$, we get 


$$
\begin{aligned}
& R C W(T)-R C W\left(T^{\prime}\right) \\
& =\sum_{u \in N_{u}, v \in D}\left[\frac{1}{d+1-d_{T}(u, v)}-\frac{1}{d+1-d_{T^{*}}(u, v)}\right] \\
& =\frac{s-1}{d+1-1}+\frac{s-1}{d+1-2}+\frac{s-1}{d+1-2}+\frac{s-1}{d+1-3}+\sum_{u \in N_{u}, v \in D \backslash K} \frac{1}{d+1-d_{T}(u, v)} \\
& -\frac{s-1}{d+1-2}-\frac{s-1}{d+1-1}-\frac{s-1}{d+1-3}-\frac{s-1}{d+1-2}-\sum_{u \in N_{u}, v \in D \backslash K} \frac{1}{d+1-d_{T^{\prime}}(u, v)} \\
& >0,
\end{aligned}
$$

and then $R C W(T)>R C W\left(T^{\prime}\right)$, this is a contradiction. Thus any vertex of $T$ outside $P(T)$ has degree at most two.

Suppose that there are at least two vertices of $T$ outside $P(T)$ with degree two. Let $y \in V(T) \backslash V(P(T))$ with $\delta_{T}(y)=2$ and let $x$ be the neighbor of $y$ which is different from $v_{\left\lfloor\frac{d}{2}\right\rfloor}$ in $T$. Let $T^{\prime \prime}$ be the tree formed from $T$ by deleting edge $y x$ and adding edge $v_{\left\lfloor\frac{d}{2}\right\rfloor} x$. Obviously, $T^{\prime \prime} \in \mathcal{N C}(n, d)$. Let $F=\left\{x, y, v_{\left\lfloor\frac{d}{2}\right]}\right\}$. Since $R C W(T)>R C W\left(T^{\prime \prime}\right)$ and $d_{T}(x, v)=d_{T^{\prime \prime}}(x, v)+1$ for $v \in V(T) \backslash F$, we get

$$
\begin{aligned}
& R C W(T)-R C W\left(T^{\prime \prime}\right) \\
= & \frac{1}{d+1-1}+\frac{1}{d+1-2}+\sum_{v \in V(T) \backslash F} \frac{1}{d+1-d_{T}(x, v)} \\
& -\frac{1}{d+1-1}-\frac{1}{d+1-2}-\sum_{v \in V(T) \backslash F} \frac{1}{d+1-d_{T^{\prime \prime}}(x, v)} \\
> & 0 .
\end{aligned}
$$

This is a contradiction. Thus there is exactly one vertex outside $P(T)$ with degree two and all other vertices of $T$ outside $P(T)$ are pendant vertices. Then, $T=N_{n, d}$.

By a direct calculation, we get

$$
\begin{aligned}
R C W\left(N_{\left.n, d, \frac{d}{2}\right]}\right]= & d+\frac{n-d+1}{d-2}+\frac{(n-d-2)(n-d-3)-2}{2(d-1)} \\
& +(n-d-1)\left(\sum_{k=1}^{\frac{d}{2}} \frac{2}{d-k}+\frac{1}{d}\right) . \text { where } d \text { is even; } \\
\left.R C W\left(N_{n, d,\left[\frac{d}{2}\right]}\right\rfloor\right)= & d+\frac{n-d-3}{d-2}+\frac{2}{d-3}+\frac{(n-d-2)(n-d-3)+4(n-d)-2}{2(d-1)} \\
& +(n-d-1)\left(\sum_{k=1}^{\frac{d-1}{2}} \frac{2}{d-k}+\frac{1}{d}\right) . \text { where } d \text { is odd. }
\end{aligned}
$$

Combining Lemmas 1 and 2, we get

Theorem 1 Let $T \in \mathbf{N C}(n, d)$, and $4 \leq d \leq n-3$. Then

$$
R C W(T) \geq R C W\left(N_{n, d}\right)
$$

with equality if and only if $T=N_{n, d}$. 
Lemma 3 For $4 \leq d \leq n-3$, there is $\operatorname{RCW}\left(N_{n, d,\left\lfloor\frac{d}{2}\right\rfloor}\right)>\operatorname{RCW}\left(N_{n, d+1,\left[\frac{d+1}{2}\right\rfloor}\right)$.

Proof. If $d$ is even, then

$$
\begin{aligned}
& R C W\left(N_{n, d, \frac{d}{2}}\right)-R C W\left(N_{n, d+1, \frac{d}{2}}\right) \\
= & \left(-1+\sum_{k=1}^{\frac{d}{2}-1} \frac{2}{d-k}\right)+\frac{5(n-d-1)}{d}-\frac{n-d-2}{d+1}-\frac{4(n-d-2)+1}{d}+\frac{n-d-1}{d-2} \\
& -\frac{n-d-3}{d-1}+\frac{(n-d-2)(n-d-3)}{2(d-1)}-\frac{(n-d-3)(n-d-4)}{2 d} \\
> & \left(\begin{array}{c}
\frac{d}{2}-1 \\
-1+\sum_{k=1}^{2} \frac{2}{d-k}
\end{array}\right)>0 .
\end{aligned}
$$

If $d$ is odd, then

$$
\begin{aligned}
& R C W\left(N_{n, d, \frac{d-1}{2}}\right)-R C W\left(N_{n, d+1, \frac{d+1}{2}}\right) \\
= & \left(-1+\sum_{k=1}^{\frac{d-1}{2}} \frac{2}{d-k}\right)+\frac{n-d-3}{d-2}-\frac{n-d-4}{d}+\frac{(n-d-2)(n-d-3)}{2(d-1)} \\
& -\frac{(n-d-3)(n-d-4)}{2 d}+\frac{n-d-1}{d-1}-\frac{n-d-2}{d+1}+\frac{2}{d-3} \\
> & \left(-1+\sum_{k=1}^{\frac{d-1}{2}} \frac{2}{d-k}\right)>0 .
\end{aligned}
$$

The result follows.

Theorem 2 For $n \geq 9$, there is

$$
R C W\left(N_{n, n-3,\left\lfloor\frac{n-3}{2}\right\rfloor}\right)<R C W\left(N_{n, n-3,\left\lfloor\frac{n-5}{2}\right\rfloor}\right)<R C W\left(N_{n, n-4,\left\lfloor\frac{n-4}{2}\right\rfloor}\right) .
$$

And $\quad R C W(T)>\operatorname{RCW}\left(N_{n, n-4,\left\lfloor\frac{n-4}{2}\right\rfloor}\right)$ for any $n$-vertex non-caterpillar $T$ different from $N_{\left.n, n-3, \mid \frac{n-3}{2}\right\rfloor}$, $N_{n, n-3,\left\lfloor\frac{n-5}{2}\right\rfloor}, \quad N_{n, n-4,\left\lfloor\frac{n-4}{2}\right\rfloor}$.

Proof. Let $T \in \mathbf{N C}(n, d)$, where $4 \leq d \leq n-3$. If $d=n-3$, then $T$ is a non-caterpillar $N_{n, n-3, i}$ where $1 \leq i \leq\left\lfloor\frac{n-3}{2}\right\rfloor$. It follows that

$$
R C W\left(N_{n, n-3, i}\right)=n-3+\frac{2}{n-3}+\frac{1}{n-4}+\sum_{k=4}^{i+3} \frac{2}{n-k}+\sum_{k=5}^{n-i} \frac{2}{n-k}+\frac{1}{n-i-4}+\frac{1}{i-1},
$$

and hence $R C W\left(N_{n, n-3, i}\right)$ is monotonically decreasing for $1 \leq i \leq\left\lfloor\frac{n-3}{2}\right\rfloor$. This implies 


$$
R C W\left(N_{n, n-3,\left[\frac{n-3}{2}\right]}\right)<R C W\left(N_{n, n-3,\left\lfloor\frac{n-5}{2}\right]}\right)<\cdots<R C W\left(N_{n, n-3,2}\right) .
$$

Now suppose that $d \leq n-4$. By Theorem 1 and Lemma 3, there is

$$
R C W(T) \geq R C W\left(N_{n, n-4,\left[\frac{n-4}{2}\right]}\right)
$$

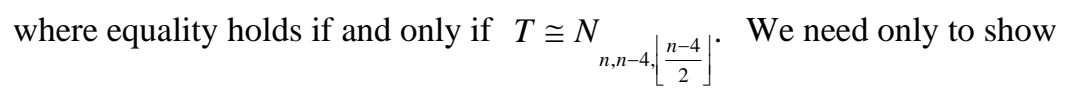

$$
R C W\left(N_{n, n-3,\left\lfloor\frac{n-5}{2}\right\rfloor}\right)<R C W\left(N_{n, n-4,\left\lfloor\frac{n-4}{2}\right]}\right) .
$$

Case 1. $n$ is odd. Let $i=\left\lfloor\frac{n-5}{2}\right\rfloor=\frac{n-5}{2}$ and $n \geq 9$. Then there is

$$
R C W\left(N_{n, n-4, \frac{n-5}{2}}\right)-R C W\left(N_{n, n-3, \frac{n-5}{2}}\right)=\left(-1+\sum_{k=6}^{\frac{n+5}{2}} \frac{2}{n-k}\right)+\frac{2}{n-5}+\frac{1}{n-6}>0 .
$$

Case 2. $n$ is even. Let $i=\left\lfloor\frac{n-5}{2}\right\rfloor=\frac{n-6}{2}$. Then there is

$$
\begin{aligned}
& R C W\left(N_{n, n-4, \frac{n-4}{2}}\right)-R C W\left(N_{n, n-3, \frac{n-6}{2}}\right) \\
& =\left(-1+\sum_{k=6}^{\frac{n+4}{2}} \frac{2}{n-k}\right)+\frac{2}{n-5}+\frac{1}{n-6}+\frac{2}{n-2}+\frac{4}{n-4}-\frac{2}{n-3}-\frac{2}{n-8} \\
& >\left(-1+\sum_{k=6}^{\frac{n+4}{2}} \frac{2}{n-k}\right)+\frac{5}{n-5}-\frac{2}{n-8} \\
& \geq 0 .
\end{aligned}
$$

Thus, the proof is finished.

\section{References}

[1] Ivanciuc, O. (2003) Graph Theory in Chemistry. In: Gasteiger, J., Ed., Handbook of Chemoinformatics, Wiley-VCH. Weinheim, 103-138. http://dx.doi.org/10.1002/9783527618279.ch6

[2] Ivanciuc, O. (2003) Topological Indices. In: Gasteiger, J., Ed., Handbook of Chemoinformatics, Wiley-VCH, Weinheim, 981-1003. http://dx.doi.org/10.1002/9783527618279.ch36

[3] Janežić, D., Miličević, A., Nikolić, S. and Trinajstić, N. (2007) Graph-Theoretical Matrices in Chemistry. University of Kragujevac, Kragujevac.

[4] Luo, W. and Zhou, B. (2009) On Ordinary and Reverse Wiener Indices of Non-Caterpillars. Mathematical and Computer Modelling, 50, 188-193. http://dx.doi.org/10.1016/j.mcm.2009.02.010

[5] Ivanciuc, O. (2000) QSAR Comparative Study of Wiener Descriptors for Weighted Molecular Graphs. Journal of Chemical Information and Modeling, 40, 1412-1422. http://dx.doi.org/10.1021/ci000068y

[6] Ivanciuc, O., Ivanciuc, T. and Balaban, A.T. (2000) The Complementary Distance Matrix, a New Molecular Graph Metric. ACH-Models in Chemistry, 137, 57-82.

[7] Ivanciuc, O., Ivanciuc, T. and Balaban, A.T. (2002) Quantitative Structure-Property Relationship Evaluation of Struc- 
tural Descriptors Derived from the Distance and Reverse Wiener Matrices. Internet Electron. Journal of ComputerAided Molecular Design, 1, 467-487.

[8] Ivanciuc, O., Ivanciuc, T. and Balaban, A.T. (1999) Vertex- and Edge-Weighted Molecular Graphs and Derived Structural Descriptors. In: Devillers, J. and Balaban, A.T., Eds., Topological Indices and Related Descriptors in QSAR and QSPR, Gordon and Breach, Amsterdam, 169-220.

[9] Cai, X. and Zhou, B. (2009) Reciprocal Complementary Wiener Numbers of Trees, Unicyclic Graphs and Bicyclic Graphs. Discrete Applied Mathematics, 157, 3046-3054. http://dx.doi.org/10.1016/j.dam.2009.05.001

[10] Zhou, B., Cai, X. and Trinajstić, N. (2009) On Reciprocal Complementary Wiener Number. Discrete Applied Mathematics, 157, 1628-1633. http://dx.doi.org/10.1016/j.dam.2008.09.010 